\title{
Alterations in Placental Gene Expression of Pregnant Women with Chronic Chagas Disease
}

Natalia A. Juiz, ${ }^{*}$ Irma Torrejón, ${ }^{\dagger}$ Marianela Burgos, ${ }^{\ddagger}$ Ana M.F. Torres, ${ }^{\dagger}$ Tomás Duffy, ${ }^{\S}$ Nelly M. Cayo, ${ }^{\dagger}$ Anahí Tabasco, * Miriam Salvo, Silvia A. Longhi, ${ }^{\star}$ and Alejandro G. Schijman*

From the Molecular Biology of Chagas Disease Laboratory, * Genetic Engineering and Molecular Biology Research Institute Dr. Héctor Torres (INGEBICONICET), Buenos Aires, Argentina; the Height Biology Institute, ${ }^{\dagger}$ National University of Jujuy, Jujuy, Argentina; the Obstetrics Service, ${ }^{\ddagger}$ Maternal and Child Department, Professor Alejandro Posadas National Hospital, Buenos Aires, Argentina; and The Scripps Research Institute, ${ }^{\S}$ University of Southern California, San Diego, California

Accepted for publication February 20, 2018.

Address correspondence to Alejandro G. Schijman, Ph.D., or Natalia A. Juiz, Ph.D., Instituto de Investigaciones en Ingeniería Genética y Biología Molecular "Dr Héctor Torres", 2490, Vuelta de Obligado, C1428ADN, Buenos Aires, Argentina. E-mail: njuiz@dna. uba.ar or schijman@dna.uba.ar.

\begin{abstract}
Trypanosoma cruzi infection in women of reproductive age is associated with congenital transmission and adverse pregnancy outcomes. The placenta is a key barrier to infection. Gene expression profiles of term placental environment from $T$. cruzi-seropositive (SP) and -seronegative (SN) mothers were characterized by RNA-Seq. Nine pools of placental RNA paired samples were used: three from SN and six from SP tissues. Each pool consisted of female/male newborns and vaginal/cesarean delivery binomials. No newborn was congenitally infected. T. cruzi satellite DNA quantitative PCR in placental tissues and maternal and neonatal blood, and parasite $18 \mathrm{~S}$ quantitative RT-PCR from placental RNA were negative, except in three SP women's bloodstream. To identify pathways associated with maternal T. cruzi infection, a gene-set association analysis was implemented: SP placental samples showed overexpression of inflammatory response and lymphocytic activation, whereas numerous biosynthetic processes were down-regulated. About 42 genes showed a significant fold-change between SP and SN groups. KISS1 and CGB5 were down-regulated, whereas KIF12, HLA-G, PRG2, TAC3, FN1, and ATXN3L were up-regulated. Several expressed genes in SP placentas encode proteins associated with preeclampsia and miscarriage. This first transcriptomics study in human term placental environment shows a placental response that may affect the fetus while protecting it from parasite infection; this host response could be responsible for the low rate of congenital transmission in chronic Chagas disease. (Am J Pathol 2018, 188: 1345-1353; https://doi.org/10.1016/j.ajpath.2018.02.011)
\end{abstract}

About 1.125 million women of fertile age are infected with Trypanosoma cruzi worldwide. ${ }^{1}$ The incidence of congenital transmission from a chronically $T$. cruzi-infected pregnant woman to her fetus is relatively low, making the passage of the parasite across the placental barrier an infrequent event. Several mechanisms exerted by the placenta to protect the fetus from the infection have been reported, such as epithelial trophoblast turnover, ${ }^{2}$ fetal production of proinflammatory cytokines, ${ }^{3}$ as well as an important role of fetal natural killer response. ${ }^{4}$

Different strategies for studying the mechanisms of placental resistance to $T$. cruzi infection have been implemented, namely, in vitro monolayer cell culture models, ex vivo infection of human chorionic villi explants, and mammal experimental models. ${ }^{5}$ However, they all have disadvantages: cell cultures cannot reflect physiological conditions; in chorionic villi explants, the immune system, essential in this process, does not participate; and placentas of experimental models are dissimilar to human placentas. ${ }^{5}$

In search of a better understanding of host-pathogen interaction in the pregnancy context, we aimed to characterize the gene expression profile of the term placental environment from $T$. cruzi-infected mothers in comparison with that of healthy noninfected mothers.

Supported by the Argentinean Ministry of Science and Technology grants PICT 2014-1188 and PICT 2015-0074, and the European Union's Research and Innovation funding program grant ERANET-LAC HD 328 (A.G.S).

Disclosures: None declared. 


\section{Materials and Methods}

\section{Ethics Statement}

The research protocols followed the tenets of the Declaration of Helsinki and Guidelines according to Resolution $\mathrm{N}^{\circ} 1480 / 11$ of the Ministerio de Salud from Argentina and were approved by the local medical ethics committees named Comité Provincial de Bioética de Jujuy and Comité de Bioética Dr. Vicente Federico del Giúdice - Hospital Nacional Prof. Alejandro Posadas. All mothers provided informed consent on their behalf before sample collection and after the purposes of the study were explained.

\section{Subjects and Samples}

Serodiagnosis of pregnant women was done by means of conventional serological methods and performed by the respective health centers based on routine assays. In maternal and umbilical cord blood samples, as well as placental tissues, presence of $T$. cruzi was tested using multiplex real-time PCR as previously described. ${ }^{6}$ Maternal infection with other pathogens that produce congenital transmission and adverse pregnancy outcome, as well as missing data or incorrect sampling, were considered as exclusion criteria.

Fresh normal placentas were obtained after labor from vaginal or cesarean deliveries and placed within 24 hours at $4^{\circ} \mathrm{C}$. Each placenta was dissected, and the middle section ${ }^{7}$ at $2 \mathrm{~cm}$ distance from the umbilical cord was isolated and placed into RNAlater solution (Applied Biosystems, Foster City, CA). Total RNA was extracted with TRIzol reagent (Invitrogen, Carlsbad, CA) and stored at $-80^{\circ} \mathrm{C}$ until used.

\section{Transcriptomic Studies}

A RNA-Seq experiment was performed in nine pools of two different placental RNA samples each: three pools $(\mathrm{C} 1, \mathrm{C} 2$, and $\mathrm{C} 3$ ) belonging to placentas from seronegative mothers (SN) and six pools (TC4 to TC9) from seropositive mothers (SP). Each pool consisted of a binomial of a female/male newborn and a vaginal/cesarean delivery. The cDNA libraries were prepared according to Illumina's TruSeq Stranded Total RNA with Ribo-Zero Gold for Human (Illumina, San Diego, CA), and a Hiseq 2.500 Illumina platform with 100-bp paired-end reads was used for sequencing. The RNA-Seq data have been submitted to the National Center for Biotechnology Information Gene Expression Omnibus (http://www.ncbi.nlm.nih.gov/geo; accession number GSE107376).

\section{RNA-Seq Data Analysis}

Raw reads were subjected to a quality test with FastQC software version 0.11.5 (Babraham Bioinformatics, Cambridge, UK; http://www.bioinformatics.babraham.ac.uk/ projects/fastqc). Sequences were mapped implementing
STAR software version $2.5,{ }^{8}$ and Picard software version 2.9.4 (Broad Institute, Cambridge, MA) tools were applied to calculate the gene expression level. Finally, the differentially expressed genes (DEGs) were identified using edgeR software version $3.6,{ }^{9}$ and principal component analysis was calculated in $\mathrm{R}$ software version 3.4.3 ( $\mathrm{R}$ Foundation for Statistical Computing, Vienna, Austria). Gene Ontology (GO) functional enrichment analysis was performed by the web-based application GOrilla (MULTIKNOWLEDGE Project; http://cbl-gorilla.cs.technion.ac.il) and gene-set association analysis for RNA-Seq with gene permutation GSAASeqGP software version 2.0. ${ }^{10}$ Gene interaction networks were analyzed by GeneMANIA software version 3.4.0 (University of Toronto, Toronto, ON, Canada; http://www.genemania.org) and visualized by the corresponding Cytoscape software version 3.0.2 plugins. ${ }^{11}$

\section{RNA-Seq Validation}

To validate the RNA-Seq results, total RNA pools used in transcriptomics and 12 independent samples (four from SN and eight from SP women) were analyzed. The cDNA was obtained implementing TaqMan Reverse Transcription Reagents (Applied Biosystems). Quantitative RT-PCR (RTqPCR) of selected DEGs were performed using 1X FastStart Universal SYBR Green Master (Roche Diagnostics Corp., Indianapolis, IN) using the following cycling conditions: $95^{\circ} \mathrm{C} 10$ minutes; 5 cycles of $95^{\circ} \mathrm{C}$ for 15 seconds and $64^{\circ} \mathrm{C} 1$ minute, 40 cycles of $95^{\circ} \mathrm{C}$ for 15 seconds, and annealing temperature as listed in Table 1, for 1 minute. The expression levels were normalized to GAPDH with primers GAPDH 5' GGTCTCCTCTGACTTCAACA-3' (forward) and GAPDH 5'-GTGAGGGTCTCTCTCTTCCT-3' (reverse).

A RT-qPCR assay targeting $T$. cruzi 18S RNA was also performed in these samples as described previously. ${ }^{12}$

Primer sequences of selected DEGs are listed in Table 1. The relative expression of each sample was calculated using the $2^{-\Delta \Delta C T}$ method with the control group as calibrator. Statistical analyses were performed using GraphPad Prism software version 6.01 (GraphPad Software, La Jolla, CA).

\section{Results}

\section{Mother/Newborn Binomials Characterization}

Sixty-five pregnant women (age range 18 to 41 years) were consecutively enrolled at Posadas Hospital, Buenos Aires, from January 2014 to November 2015 and at Jujuy Maternal Hospital from January to September 2016 (Figure 1). Sixteen were $\mathrm{SN}$ and $49 \mathrm{SP}$, but 24 of these binomials were omitted because of exclusion criteria. Numbers of vaginal and cesarean deliveries and also numbers of female/male newborns in each case are detailed in Figure 1. All newborn umbilical cord and peripheral blood samples were collected during the first hour of life and had nondetectable $T$. cruzi satellite DNA by PCR, in 
Table 1 Oligonucleotides Used in RNA-Seq Validation

\begin{tabular}{|c|c|c|c|c|}
\hline Gene & Forward & Reverse & $\mathrm{Ta},{ }^{\circ} \mathrm{C}$ & Amplicon length, bp \\
\hline$H L A-G$ & 5'-CTGGTTGTCCTTGCAGCTGTAG- 3' & 5'-ССТTTTCAATCTGAGCTCTTCTTTCT-3' & 57 & 80 \\
\hline FN1 & 5'-CAAACACTAATGTTAATTGCCCA-3' & 5'-GGTCTGCTAACATCACTCCA-3' & 56 & 156 \\
\hline ATXN3L & 5'-AAGATGAACATCTCCGCAGT-3' & 5'-GGCCTTTCGTGTAGGTATGA-3' & 56 & 214 \\
\hline TAC3 & 5'-GGATCATGCTGCTATTCACAG-3' & 5'-ATGCATGTCACGTTTCTCGG-3' & 56 & 245 \\
\hline CGB5 & 5'-CAGCATCCTACAACCTCCTG-3' & $5^{\prime}-$ CGCGGGTCATGGTGG-3' & 67 & 277 \\
\hline KISS1 & 5'-GGACCTGCCTCTTCTCACCA-3' & 5'-АTTCTAGCTGCTGGCCTGTG-3' & 54 & 140 \\
\hline
\end{tabular}

Primer sequences of selected DEGs, annealing temperatures of RT-qPCR procedures ( $\mathrm{Ta}$ ) and amplicons length.

agreement with microhematocrit findings. Placental tissues were also real-time quantitative PCR negative (Table 2).

For RNA-Seq studies, only term pregnancies with times of gestation above 37 weeks (mean $=39.22 \pm 1.15$ weeks) were selected. Of 29 placental samples from SP, 12 were selected, grouped into six pools, and of 12 samples from $\mathrm{SN}, 6$ were selected, grouped into three pools. The construction of pools took into account a balance (1:1) of newborn sex and mode of delivery variables, because they could become confounding factors (Table 2). These samples belonged to mothers with a mean age of $27.92 \pm 7.55$ and $27.67 \pm 7.55$ years, in the SP and SN groups, respectively $(P>0.05)$. No significant differences were found in time of gestation between the SP and SN groups (Table 2) $(P=0.597)$.

Of SP binomials, three pools were prepared with samples from women with positive bloodstream PCR results (TC4, TC5, and TC6) and another three with nondetectable parasite DNA in maternal peripheral blood after admission to maternity service (TC7, TC8, and TC9). Moreover, none of the placental tissues were positive for the determination of 18s RNA for T. cruzi (Table 2).

\section{Relatedness among Gene Expression Patterns of Pools}

First, principal component analysis was performed for a preliminary clustering of samples on the basis of complete gene expression data without prior statistical filtering (Figure 2). The first two principal components, which explain $86 \%$ (PC1 61\% and PC2 25\%) of the variance among samples, separate pools of $\mathrm{SN}(\mathrm{C} 1, \mathrm{C} 2$, and $\mathrm{C} 3)$ from those of SP (TC4 to TC9) mothers. However, the gene expression observed in the SP group could not differentiate between mothers with detectable or nondetectable $T$. cruzi DNA in peripheral blood close to delivery.

\section{Differentially Expressed Genes}

Only 42 genes were differentially expressed with a significance level $<0.05$ and a fold change $|\mathrm{FC}|>1.5$ and 26 with $|\mathrm{FC}|>2$, comparing SN and SP placentas (Table 3). The most represented transcript in SP group placentas was KIF12, a kinesin superfamily member with a role in microtubule cytoskeleton. Another interesting gene that turned out to be more expressed in the SP group was

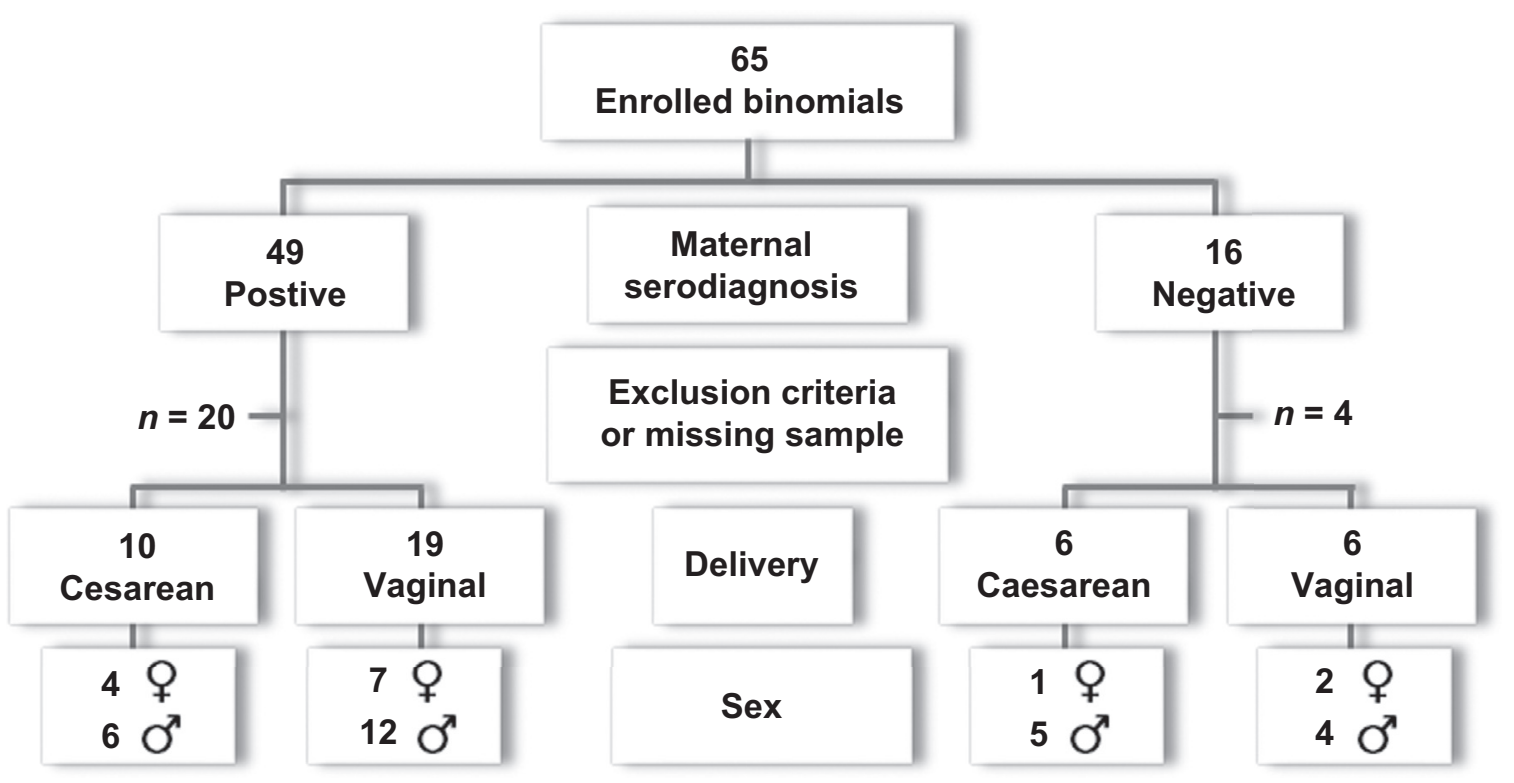

Figure 1 Flowchart of enrolled mother/newborn binomials in the prospective study. 
Table 2 Characteristics of Mother/Newborn Binomials under Study

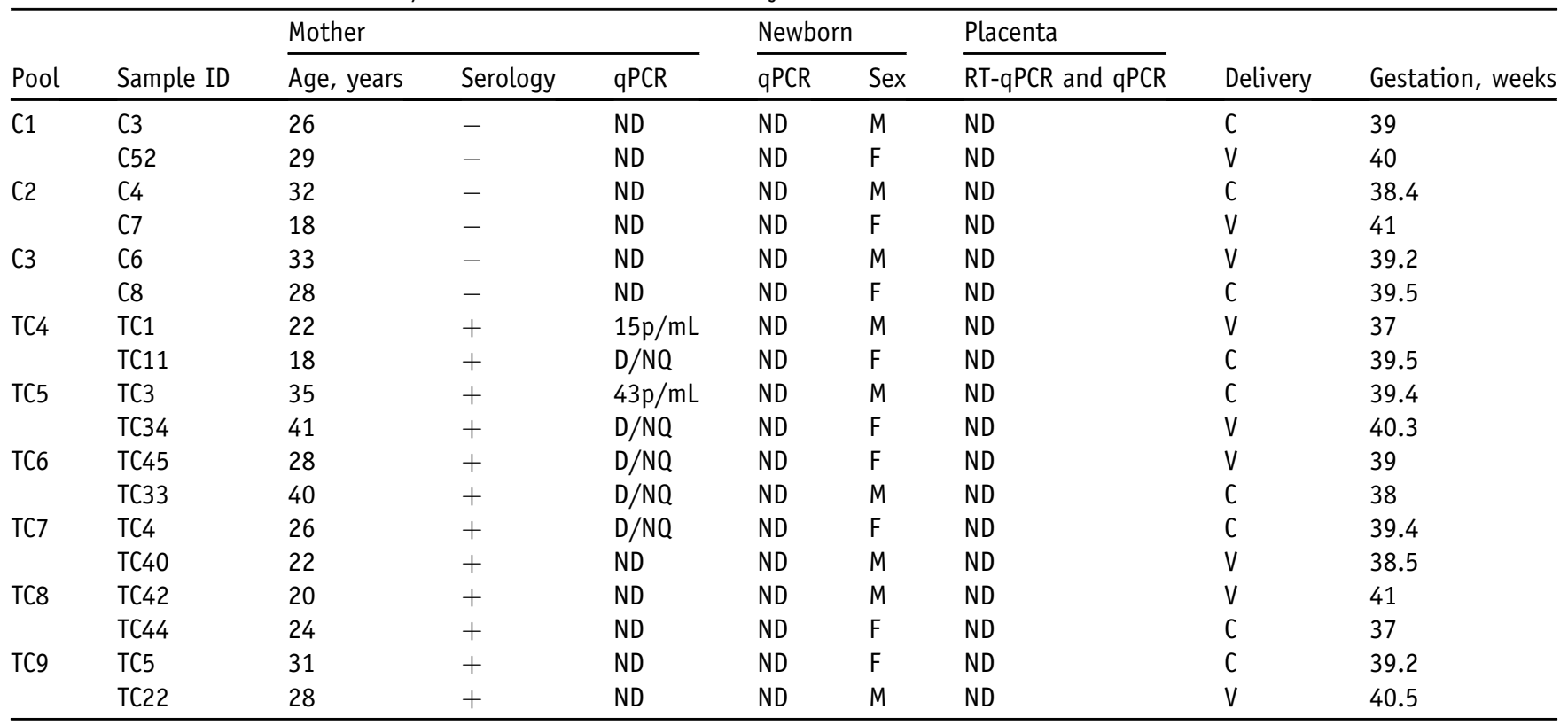

Maternal age and serological diagnosis, newborn sex and results of $T$. cruzi satellite DNA qPCR expressed as parasites per milliliter (p/mL) in maternal and neonatal blood, as well as T. cruzi satellite DNA qPCR and 18S RNA-based RT-qPCR in placental tissues. It is also indicated delivery mode and time of gestation.

F, female; M, male; C, cesarean; D/NQ, detectable but nonquantifiable (parasitic load below $1.5 \mathrm{p} / \mathrm{mL}$; [6]); ND, nondetectable; RT-qPCR, quantitative RTPCR; qPCR, real-time quantitative PCR; $V$, vaginal.

$H L A-G$. It belongs to the HLA class I that is expressed on fetal-derived placental cells and is related to immune tolerance in pregnancy. In addition, $P R G 2$, a cytotoxin involved in antiparasitic defense mechanisms, MUC4, a mucin that provides protection to epithelial cells, and $T A C 3$, the protein of which is expressed in the syncytiotrophoblast, had also a greater transcription level in the SP group.

There was also a down-regulation of immunomodulatory activity genes in the SP group, such as ILIFIO and KISS1, inhibitors of chemotaxis and invasion, with a putative role in cell-matrix adhesion and that stimulate the secretion of gonadotropin. Related to the latter, the gene encoding the beta subunit of chorionic gonadotropin, $C G B 5$, was also down-regulated, whereas fibronectine 1 $(F N 1)$, presents in cell surface and the extracellular matrix, was overexpressed in placentas from SP mothers. Finally, the most down-regulated gene was MIR6723, a microRNA that may be involved in post-transcriptional regulation.

\section{Functional Pathway Analysis}

To identify pathways significantly associated with the SP group in the placental environment, we first used the GOrilla tool for identifying enriched GO terms, considering genes with an adjusted $P<0.05$ (Supplemental Table S1). Extracellular matrix organization emerged from positive FC genes, whereas pathways related to exocytosis, neutrophil degranulation, and glutamine and glutathione biosynthetic processes emerged from the list of negative FC genes.

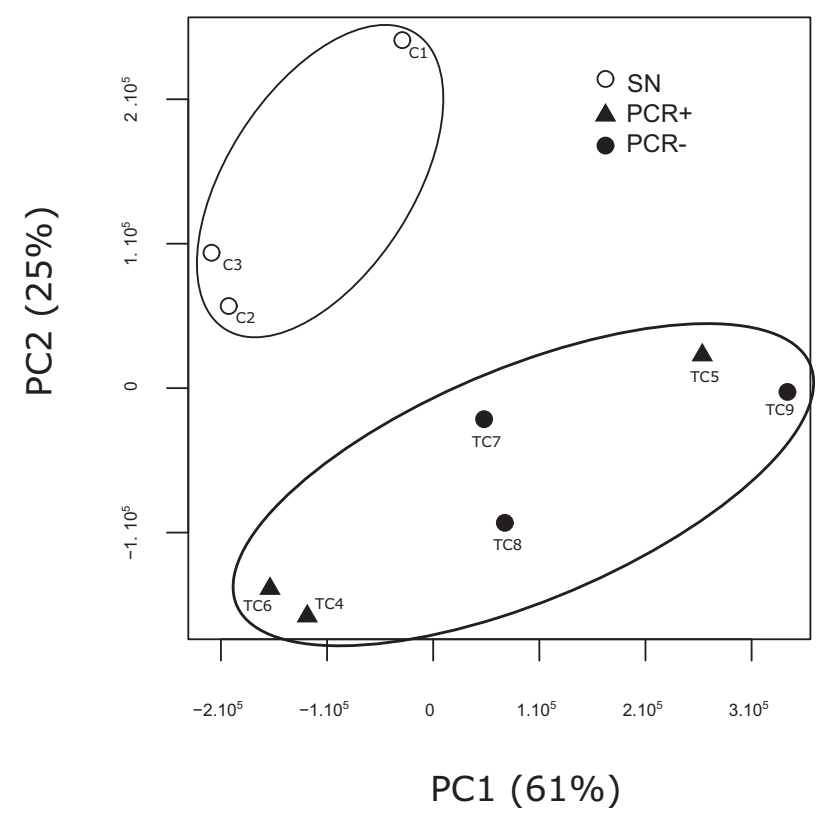

Figure 2 Principal component analysis (PCA). PCA plot of human placental transcriptome from pooled samples of seronegative $(\mathrm{SN})$ and seropositive (SP) mothers with detectable (PCR+) or nondetectable (PCR-) T. cruzi DNA in peripheral blood at the time of delivery. Circles cluster SN and SP samples. The $x$ and $y$ axes correspond to the first (PC1) and second (PC2) principal components, respectively, with their explained variances. 
Table 3 List of Differentially Expressed Genes Detected by RNA-Seq Analysis

\begin{tabular}{|c|c|c|c|c|c|c|c|}
\hline \multicolumn{4}{|c|}{ Up-regulated DEGs } & \multicolumn{4}{|c|}{ Down-regulated DEGs } \\
\hline Locus* & Gene & FC & $\mathrm{P}_{\mathrm{adj}}$ & Locus & Gene & $\mathrm{FC}$ & Padj \\
\hline NM_138424 & KIF12 & 6.36 & 0.040 & NR_106781 & MIR6723 & -4.80 & 0.000 \\
\hline NR_046087 & GS1 & 5.80 & 0.040 & NR_033557 & LOC100422737 & -3.71 & 0.033 \\
\hline NM_001135995 & ATXN3L & 5.77 & 0.028 & NM_020672 & S100A14 & -3.16 & 0.003 \\
\hline NR_108087 & LINC01087 & 3.72 & 0.038 & NM_058173 & MUCL1 & -2.82 & 0.004 \\
\hline NM_002127 & $H L A-G$ & 3.29 & 0.000 & NM_033043 & CGB5 & -2.37 & 0.033 \\
\hline NM_022073 & EGLN3 & 3.17 & 0.003 & NM_173161 & IL1F10 & -2.30 & 0.011 \\
\hline NM_002728 & PRG2 & 3.07 & 0.000 & NM_001191321 & GABRB3 & -2.27 & 0.000 \\
\hline NM_001306094 & NPIPB15 & 2.88 & 0.003 & NM_000771 & CYP2C9 & -1.99 & 0.037 \\
\hline NM_018406 & MUC4 & 2.48 & 0.021 & NM_014400 & LYPD3 & -1.95 & 0.000 \\
\hline NR_026758 & FAR2P1 & 1.77 & 0.033 & NM_024807 & TREML2 & -1.53 & 0.033 \\
\hline NR_033968 & GUSBP9 & 1.76 & 0.033 & NM_018643 & TREM1 & -1.51 & 0.001 \\
\hline NM_152666 & PLD5 & 1.75 & 0.039 & & & & \\
\hline NM_001447 & FAT2 & 1.66 & 0.010 & & & & \\
\hline NR_003263 & SDHAP3 & 1.64 & 0.034 & & & & \\
\hline NM_003670 & BHLHE40 & 1.61 & 0.039 & & & & \\
\hline NM_000887 & ITGAX & 1.59 & 0.010 & & & & \\
\hline NM_001083538 & POTEE & 1.58 & 0.010 & & & & \\
\hline
\end{tabular}

Genes with fold change $|\mathrm{FC}|>1.5$ and adjusted $P<0.05$, detected after comparing placental samples from SN and SP pregnant women.

*Loci are available from the National Center for Biotechnology Information (https://www.ncbi.nlm.nih.gov).

A gene-set association analysis was also performed by GSAA-Seq, a cutoff-free strategy of genome-wide patterns in the measured gene expression. In this approach, choosing arbitrary cutoffs is avoided, and accumulative subtle changes in gene expression within the same gene set are identified. The resulting gene sets significantly associated with a false discovery rate $q$ value $<25 \%$ and nominal $P<5 \%$ were represented in an enrichment map (Figure 3). Several pathways were overexpressed in the SP compared with SN placentas; some of them were related to immune response, cytoskeleton and calcium transport, cell cycle, DNA synthesis, and chromatin modifications. On the other hand, processes such as biosynthesis and respiratory chain, vesicle transport, and metabolism of lipids and hormones were down-regulated, as were networks associated with protein metabolism (synthesis and proteolysis by proteasome).

Within the top $10 \mathrm{GO}$ pathways of the ranked list of over-represented genes from SP, which is based on the false discovery rate q values and on the Gene Set Normalized Association Score, which reflects how much a pathway is over- or under-represented, five pathways were related to calcium transport, and three to immune response (Supplemental Table S2). Interestingly, the fourth in this list was the GO Positive Regulation of Acute Inflammatory Response with 16 (57\%) genes contributing to the enrichment score. In the eighth and tenth positions, there were T-cell Selection and Positive Regulation of Neutrophil Migration networks.
Regarding gene sets that were under-represented in placentas of SP mothers, several pathways related to respiratory chain were on the top of the list (Supplemental Table S2): Mitochondrial Respiratory Chain Complex I Biogenesis and Energy Derivation By Oxidation of Organic Compounds. Moreover, translation processes were also represented in the first positions: Translational Initiation and Translational Termination. Lastly, processes of vesicle transport were also affected by maternal infection, for example, Establishment of Protein Localization to Endoplasmic Reticulum (Supplemental Table S2).

\section{RNA-Seq Validation}

To validate the RNA-Seq data using RT-qPCR, seven DEGs were selected on the basis of their high FCs and low $P$ values: FN1, ATXN3L, TAC3, CGB5 and HLA-G, PRG2, and KISS1. The expression patterns of these genes are shown in Figure 4. The results showed that the expression patterns as determined by RT-qPCR were consistent with those obtained by RNA-Seq $(P>0.05)$, confirming the accuracy of the RNA-Seq results in this study.

\section{Discussion}

Congenital T. cruzi infection remains a global health problem with an estimation of 8700 cases per year in endemic 


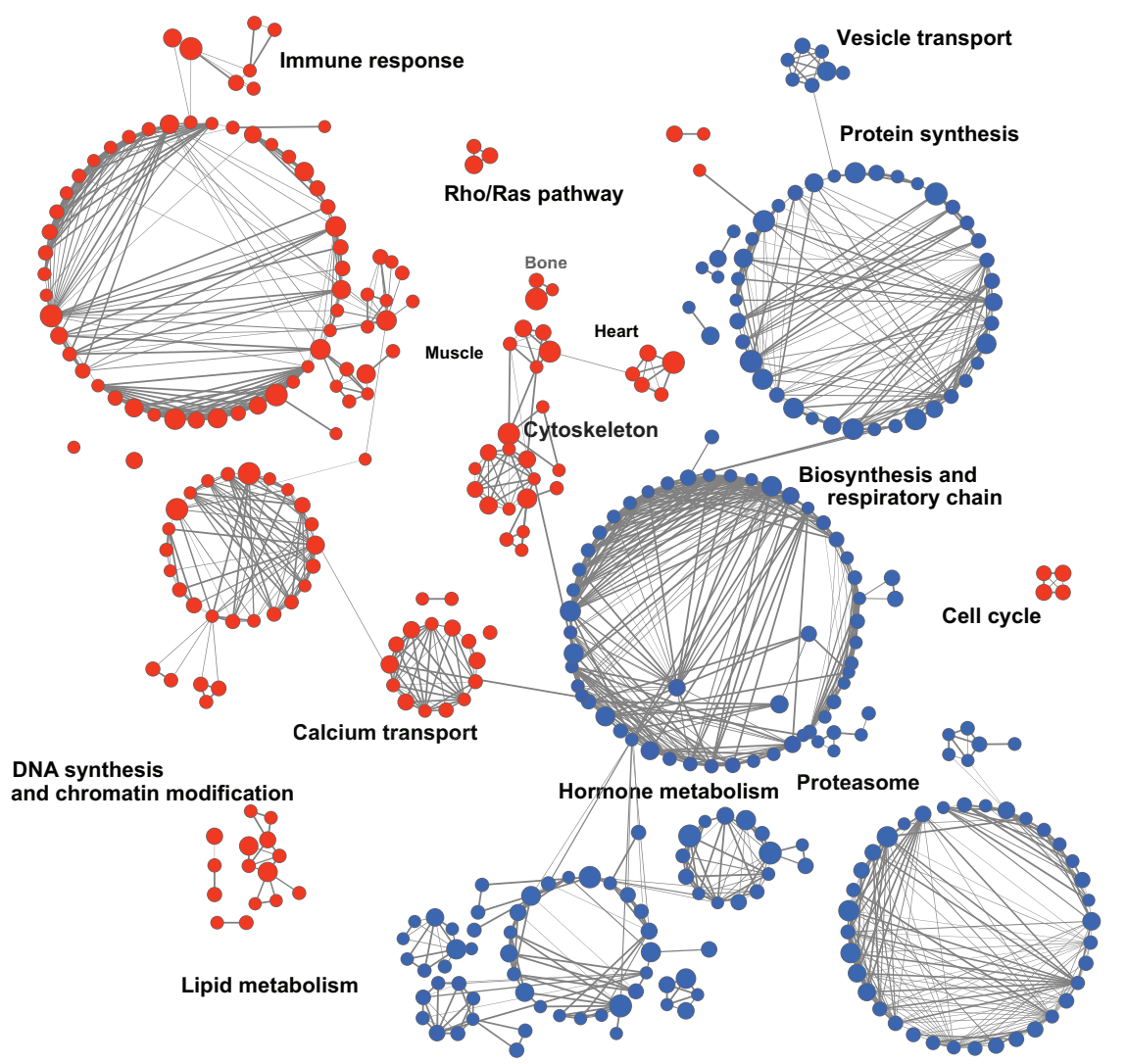

Figure 3 Gene-set association analysis. Representation of biological pathways with false discovery rate $q$ value $<25 \%$ and nominal $P<5 \%$. Nodes correspond to gene sets, where red nodes correspond to up-regulated gene-sets and nodes in blue correspond to down-regulated ones, and links correspond to overlap of member genes. To improve layout, the networks were manually rearranged. Major clusters were manually labeled. countries. ${ }^{1}$ Maternal parasitic loads and strain placental tropism have been implicated in the likelihood of congenital transmission, which is an infrequent event in chronic Chagas disease pregnancy. ${ }^{13,14}$ Indeed, trypanocidal treatment of women at reproductive age reduces the rate of vertical transmission. ${ }^{15}$

Family clustering and congenital transmission in second generations in nonendemic areas contribute to perpetuate Chagas disease. ${ }^{14,16}$ A case of a triplet born to a Chagas disease mother from a triamniotic dichorionic delivery was described by Burgos et al, ${ }^{17}$ who reported the occurrence of congenital infection in both sisters sharing one placenta and no infection in the third one, indicative of the protective role of the placenta. Human genomic polymorphism in placental genes has been also associated to the occurrence of transmission. ${ }^{18}$ Anti-T. cruzi seropositivity in mothers was associated with a history of adverse reproductive outcomes. ${ }^{19}$ Nevertheless, there is still a knowledge gap regarding the effect of the parasite infection in pregnancy outcome.

\section{Placental Gene Expression Profile in SP Women}

In the present study, even though a few genes $(N=42)$ had a significant FC between SP and SN groups, the deeper gene-set association analysis of the RNA-Seq data suggests the existence of a gene expression profile in the chorionic villi region of placentas from $T$. cruzi-seropositive mothers that differs from that of placentas from uninfected women, as revealed by principal component analysis.

This first study in human placental environment is consistent with our previous findings in placental gene expression from mice infected with $T$. cruzi compared with noninfected dams. ${ }^{12}$ Indeed, the analysis showed several biosynthetic processes down-regulated in both murine and human placental studies, including lipid and protein metabolisms, whereas immunological gene sets were enriched in infected dams and mothers. In fact, in both studies, the inflammatory response and lymphocytic activation were overexpressed in placental tissues from infected groups. It has been already reported that newborns of $T$. cruzi-infected mothers are prone to produce higher levels of proinflammatory cytokines in comparison with those born to noninfected mothers, and there is increasing evidence suggesting that maternal infection during pregnancy affects the developing immune system of fetuses independent of the vertical transmission of pathogens. ${ }^{20}$ Nevertheless, the placenta could reduce the damage produced by the inflammatory response by decreasing the exocytosis process, including neutrophil degranulation, because we have observed a significantly lower expression in related genes in the SP group. These results are also consistent with those from mice placenta, where genes associated with the secretory granule were down-regulated. ${ }^{12}$ Moreover, vesicle 


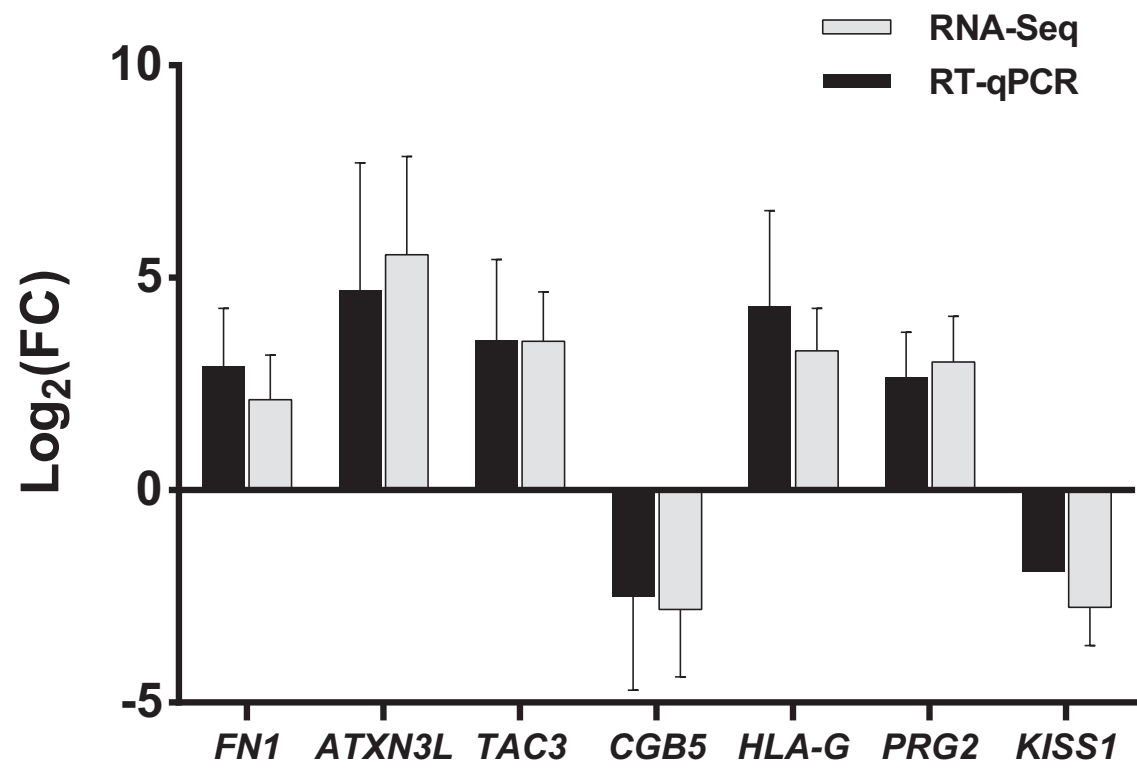

Figure 4 RT-qPCR validation analysis of gene expression. Bars represent the mean gene expression levels in seropositive (SP) relative to seronegative (SN) samples, and vertical lines show $\mathrm{SD}$. Data are expressed as means \pm SD. transport processes also decreased in placentas from SP women and infected mice.

Because inflammatory activity leads to extracellular matrix damage, it is not surprising that we have found an activation of extracellular matrix organization-related genes. Previous studies had reported the importance of the extracellular matrix remodeling and that fibronectin was not affected at a protein level ${ }^{21}$; however, our findings suggest that the placenta produces de novo synthesis of this protein, as well as collagen, during $T$. cruzi infection.

This differential gene expression in SP placentas was not associated with the presence of parasitic DNA or RNA in the tissues subjected to RNA-Seq analysis. Nevertheless, real-time quantitative PCR and RT-qPCR analysis in in-term placentas do not reflect the dynamics of placental parasite burden during pregnancy. Indeed, our transcriptomic analysis represents a placental terminal stage.

\section{Differentially Expressed Genes in Placentas from SP Women}

Infection with $T$. cruzi during pregnancy could result in low birth weight, still birth, spontaneous abortion, growth restriction, intrauterine fetal death, and fetal abnormalities. ${ }^{22}$ There are some studies reporting similar or slightly higher prevalence of abortions and stillbirths in $T$. cruzi-seropositive mothers than in uninfected mothers, and there are also some contradictions about the consequences on low birth weight. ${ }^{23}$ In addition, if that effect really exists it, is not clear whether it is a result of congenital infection or placental dysfunction. ${ }^{24}$

It is worth noting that in this study, SP human placentas showed a down-regulation of the KISS1 gene, which encodes kisspeptin, low serum levels of which have been related to preeclampsia and intrauterine growth restriction. $^{25}$ Moreover, KISS1 low expression levels have been associated with recurrent pregnancy loss, ${ }^{26}$ and low serum levels of its protein, as well as of human chorionic gonadotropin (hCG), have been proposed as markers of miscarriage. ${ }^{27}$ Interestingly, the gene $C G B 5$ that encodes the beta 5 subunit of hCG was down-regulated in SP human placentas.

Moreover, the DEG TAC3 had a higher transcription activity in SP placentas. Its coding protein, neurokinin B $(\mathrm{NKB})$, is expressed by syncytiotrophoblasts, and its overexpression has been also associated with preeclampsia. ${ }^{28}$ Both, kisspeptin and NKB play a key role in the placental expression of hCG in response to estradiol. ${ }^{29}$ Furthermore, PRG2 gene coding protein was also up-regulated in SP placentas. It encodes the preform of eosinophil major basic protein (proMBP), the predominant constituent of eosinophil granules, which have been associated with $T$. cruzi clearance through extracellular killing in the absence of antibodies. $^{30} \mathrm{MBP}$ is present in placenta inhibiting the pregnancy-associated plasma protein A (PAPPA). ${ }^{31}$ Low levels of PAPPA are related to intrauterine growth restriction, ${ }^{32}$ so the higher transcription of its inhibitor observed in SP placentas might play a role in pregnancy outcome. Both PAPPA and hCG low levels have been proposed as biomarkers of preeclampsia. ${ }^{33}$ The previously mentioned inflammatory response due to maternal infection has also been associated with preeclampsia. ${ }^{34}$ Finally, GOrilla analysis allowed detection of altered glutamine and glutathione biosynthetic processes, consistently with a recent proteomics analysis of human placenta, which showed that, not only inflammatory response, but also glutathione metabolism dysfunction may contribute to preeclampsia. ${ }^{35}$ Taken together, it is tempting to speculate that mothers with Chagas disease could be more prone to manifest 
preeclampsia than noninfected ones, which can be worth studying. This would be of relevance in chronic Chagas disease, because preeclampsia is associated with an increase of future heart failure and cardiovascular diseases. ${ }^{36}$ However, there are no studies exploring the role of $T$. cruzi infection in development of preeclampsia, such as has been observed in placental malaria ${ }^{37}$ and other parasitic infections. ${ }^{38,39}$

Another DEG was $H L A-G$ with a positive $\mathrm{FC}$, an immunomodulatory molecule involved in generating tolerance during pregnancy. A study of malaria showed that higher levels of HLA-G in infected mothers correlated with higher HLA-G levels in their newborns and infants; this phenomenon was related to a higher susceptibility of Plasmodium infection in children belonging to binomials with high HLA-G serum levels. ${ }^{40}$ It would be of interest to study this phenomenon in the context of $T$. cruzi infection.

This is the first placental transcriptomics study in natural human infection, which may contribute to the understanding of placental mechanisms induced by maternal $T$. cruzi infection. Our results suggest that although most fetuses from infected mothers remain uninfected, they may be affected by the placental response that protects them from the parasite.

\section{Acknowledgments}

We thank Paula Beati (INGEBI-CONICET), Leandro Simonetti (INGEBI-CONICET), and Carlos David Bruque (ANLIS-MALBRAN) for their bioinformatics contributions.

\section{Supplemental Data}

Supplemental material for this article can be found at https://doi.org/10.1016/j.ajpath.2018.02.011.

\section{References}

1. WHO: Integrating Neglected Tropical Diseases into Global Health and Development: Fourth WHO Report on Neglected Tropical Diseases. Geneva, Switzerland, World Health Organization, 2017

2. Liempi A, Castillo C, Carrillo I, Muñoz L, Droguett D, Galanti N, Maya JD, Kemmerling U: A local innate immune response against Trypanosoma cruzi in the human placenta: the epithelial turnover of the trophoblast. Microb Pathog 2016, 99:123-129

3. Cuna WR, Choque AG, Passera R, Rodriguez C: Pro-inflammatory cytokine production in chagasic mothers and their uninfected newborns. J Parasitol 2009, 95:891-894

4. Hermann E, Alonso-Vega C, Berthe A, Truyens C, Flores A, Cordova M, Moretta L, Torrico F, Braud V, Carlier Y: Human congenital infection with Trypanosoma cruzi induces phenotypic and functional modifications of cord blood NK cells. Pediatr Res 2006, 60:38-43

5. Fretes RE, Kemmerling U: Mechanism of Trypanosoma cruzi placenta invasion and infection: the use of human chorionic villi explants. J Trop Med 2012, 2012:614820

6. Duffy T, Cura CI, Ramirez JC, Abate T, Cayo NM, Parrado R, Bello ZD, Velazquez E, Muñoz-Calderon A, Juiz NA, Basile J, Garcia L, Riarte A, Nasser JR, Ocampo SB, Yadon ZE, Torrico F, de Noya BA, Ribeiro I, Schijman AG: Analytical performance of a multiplex real-time PCR assay using TaqMan probes for quantification of Trypanosoma cruzi satellite DNA in blood samples. PLoS Negl Trop Dis 2013, 7:e2000

7. Sood R, Zehnder JL, Druzin ML, Brown PO: Gene expression patterns in human placenta. Proc Natl Acad Sci U S A 2006, 103:5478-5483

8. Dobin A, Gingeras TR: Mapping RNA-seq reads with STAR. Curr Protoc Bioinformatics 2015, 51:11.14.1-11.14.19

9. Robinson MD, McCarthy DJ, Smyth GK: edgeR: a Bioconductor package for differential expression analysis of digital gene expression data. Bioinformatics 2010, 26:139-140

10. Xiong Q, Mukherjee S, Furey TS: GSAASeqSP: a toolset for gene set association analysis of RNA-Seq data. Sci Rep 2014, 4:6347

11. Merico D, Isserlin R, Stueker O, Emili A, Bader GD: Enrichment map: a network-based method for gene-set enrichment visualization and interpretation. PLoS One 2010, 5:e13984

12. Juiz NA, Solana ME, Acevedo GR, Benatar AF, Ramirez JC, da Costa PA, Macedo AM, Longhi SA, Schijman AG: Different genotypes of Trypanosoma cruzi produce distinctive placental environment genetic response in chronic experimental infection. PLoS Negl Trop Dis 2017, 11:e005436

13. Bua J, Volta BJ, Velazquez EB, Ruiz AM, De Rissio AM, Cardoni RL: Vertical transmission of Trypanosoma cruzi infection: quantification of parasite burden in mothers and their children by parasite DNA amplification. Trans R Soc Trop Med Hyg 2012, 106:623-628

14. Bisio M, Seidenstein ME, Burgos JM, Ballering G, Risso M, Pontoriero R, Moreau M, Altcheh J, Leguizamón MS, Freilij H, Marceillac M, Schijman AG: Urbanization of congenital transmission of Trypanosoma cruzi: prospective polymerase chain reaction study in pregnancy. Trans R Soc Trop Med Hyg 2011, 105:543-549

15. Messenger LA, Gilman RH, Verastegui M, Galdos-Cardenas G, Sanchez G, Valencia E, Sanchez L, Malaga E, Rendell VR, Jois M, Shah V, Santos N, Abastoflor MDC, LaFuente C, Colanzi R, Bozo R, Bern C; Working Group on Chagas Disease in Bolivia and Peru: Toward improving early diagnosis of congenital Chagas disease in an endemic setting. Clin Infect Dis 2017, 65:268-275

16. Sánchez Negrette O, Mora MC, Basombrío MA: High prevalence of congenital Trypanosoma cruzi infection and family clustering in Salta, Argentina. Pediatrics 2005, 115:e668-e672

17. Burgos JM, Altcheh J, Petrucelli N, Bisio M, Levin MJ, Freilij H, Schijman AG: Molecular diagnosis and treatment monitoring of congenital transmission of Trypanosoma cruzi to twins of a triplet delivery. Diagn Microbiol Infect Dis 2009, 65:58-61

18. Juiz NA, Cayo NM, Burgos M, Salvo ME, Nasser JR, Búa J, Longhi SA, Schijman AG: Human polymorphisms in placentally expressed genes and their association with susceptibility to congenital Trypanosoma cruzi infection. J Infect Dis 2016, 213:1299-1306

19. Gamboa-León R, Ramirez-Gonzalez C, Pacheco-Tucuch FS, O’Shea M, Rosecrans K, Pippitt J, Dumonteil E, Buekens P: Seroprevalence of Trypanosoma cruzi among mothers and children in rural Mayan communities and associated reproductive outcomes. Am J Trop Med Hyg 2014, 91:348-353

20. Dauby N, Goetghebuer T, Kollmann TR, Levy J, Marchant A: Uninfected but not unaffected: chronic maternal infections during pregnancy, fetal immunity, and susceptibility to postnatal infections. Lancet Infect Dis 2012, 12:330-340

21. Duaso J, Yanez E, Castillo C, Galanti N, Cabrera G, Corral G, Maya JD, Zulantay I, Apt W, Kemmerling U: Reorganization of extracellular matrix in placentas from women with asymptomatic Chagas disease: mechanism of parasite invasion or local placental defense? J Trop Med 2012, 2012:758357

22. Nourollahpour Shiadeh M, Behboodi Moghadam Z, Adam I, Saber V, Bagheri M, Rostami A: Human infectious diseases and risk of preeclampsia: an updated review of the literature. Infection 2017, 45:589-600

23. Torrico F, Alonso-Vega C, Suarez E, Rodriguez P, Torrico MC, Dramaix M, Truyens C, Carlier Y: Maternal Trypanosoma cruzi infection, pregnancy outcome, morbidity, and mortality of congenitally 
infected and non-infected newborns in Bolivia. Am J Trop Med Hyg 2004, 70:201-209

24. Carlier Y, Truyens C: Congenital Chagas disease as an ecological model of interactions between Trypanosoma cruzi parasites, pregnant women, placenta and fetuses. Acta Trop 2015, 151:103-115

25. Armstrong RA, Reynolds RM, Leask R, Shearing CH, Calder AA, Riley SC: Decreased serum levels of kisspeptin in early pregnancy are associated with intra-uterine growth restriction and pre-eclampsia. Prenat Diagn 2009, 29:982-985

26. Park DW, Lee SK, Hong SR, Han AR, Kwak-Kim J, Yang KM: Expression of Kisspeptin and its receptor GPR54 in the first trimester trophoblast of women with recurrent pregnancy loss. Am J Reprod Immunol 2012, 67:132-139

27. Jayasena CN, Abbara A, Izzi-Engbeaya C, Comninos AN, Harvey RA, Gonzalez Maffe J, Sarang Z, Ganiyu-Dada Z, Padilha AI, Dhanjal M, Williamson C, Regan L, Ghatei MA, Bloom SR, Dhillo WS: Reduced levels of plasma kisspeptin during the antenatal booking visit are associated with increased risk of miscarriage. J Clin Endocrinol Metab 2014, 99:E2652-E2660

28. Page NM, Woods RJ, Gardiner SM, Lomthaisong K, Gladwell RT, Butlin DJ, Manyonda IT, Lowry PJ: Excessive placental secretion of neurokinin B during the third trimester causes pre-eclampsia. Nature 2000, 405:797-800

29. Oride A, Kanasaki H, Mijiddorj T, Sukhbaatar U, Ishihara T, Kyo S: Regulation of kisspeptin and gonadotropin-releasing hormone expression in rat placenta: study using primary cultures of rat placental cells. Reprod Biol Endocrinol 2015, 13:90

30. Nakhle MC, de Menezes Mda C, Irulegui I: Eosinophil levels in the acute phase of experimental Chagas' disease. Rev Inst Med Trop Sao Paulo 1989, 31:384-391

31. Weyer K, Glerup S: Placental regulation of peptide hormone and growth factor activity by proMBP. Biol Reprod 2011, 84:1077-1086

32. Albu A, Anca A, Horhoianu V, Horhoianu I: Predictive factors for intrauterine growth restriction. J Med Life 2014, 7:165-171
33. Paredes V, Espinoza-Caicedo JA, Salazar-Pousada D, Escobar GS, Pérez-López FR, Chedraui P: Lower placental growth factor and higher free $\beta$-hCG and PAPP-A levels in the fetal circulation of nearterm pregnancies complicated with severe preeclampsia. Gynecol Endocrinol 2017, 33:79-81

34. Rustveld LO, Kelsey SF, Sharma R: Association between maternal infections and preeclampsia: a systematic review of epidemiologic studies. Matern Child Health J 2008, 12:223-242

35. Jin X, Xu Z, Cao J, Shao P, Zhou M, Qin Z, Liu Y, Yu F, Zhou X, Ji W, Cai W, Ma Y, Wang C, Shan N, Yang N, Chen X, Li Y: Proteomics analysis of human placenta reveals glutathione metabolism dysfunction as the underlying pathogenesis for preeclampsia. Biochim Biophys Acta 2017, 1865:1207-1214

36. Wu P, Haththotuwa R, Kwok CS, Babu A, Kotronias RA, Rushton C, Zaman A, Fryer AA, Kadam U, Chew-Graham CA, Mamas MA: Preeclampsia and future cardiovascular health: a systematic review and meta-analysis. Circ Cardiovasc Qual Outcomes 2017, 10:e003497

37. Gueneuc A, Deloron P, Bertin GI: Usefulness of a biomarker to identify placental dysfunction in the context of malaria. Malar J 2017, $16: 11$

38. Alvarado-Esquivel C, Vázquez-Alaníz F, Sandoval-Carrillo AA, SalasPacheco JM, Hernández-Tinoco J, Sánchez-Anguiano LF, Liesenfeld O: Lack of association between Toxoplasma gondii infection and hypertensive disorders in pregnancy: a case-control study in a Northern Mexican population. Parasit Vectors 2014, 7:167

39. Soydinc HE, Kan I, Dal T, Evsen MS, Sak ME, Ozler A, Turgut A, Yildiz I: Evaluation of the relationship between preeclampsia and seropositivity of infectious disease in maternal plasma. Clin Ter 2013, 164:e199-e202

40. Sadissou I, d'Almeida T, Cottrell G, Luty A, Krawice-Radanne I, Massougbodji A, Moreau P, Moutairou K, Garcia A, Favier B, RouasFreiss N, Courtin D: High plasma levels of HLA-G are associated with low birth weight and with an increased risk of malaria in infancy. Malar J 2014, 13:312 